\title{
Based on COVID-19, Analysis of the development strategy of China's self-operated e-commerce --JD.com as an example
}

\author{
Guiyang Wang* \\ Business School University of Western Australia 35 Stirling Hwy, Crawley WA 6009 Australia
}

*Corresponding author: 23230356@student.uwa.edu.au

\begin{abstract}
With the development of China's economy, more and more Chinese companies are developing and growing out of China to connect with the world. In the field of e-commerce industry, a lot of research has been conducted by scholars. However, the research on self-operated ecommerce in the context of the COVID-19 is still a blank. As the largest self-operated e-commerce platform in China, JD's development is significantly representative for the whole field. This paper focuses on SWOT analysis combined with specific data and the current situation of the e-commerce industry to analyze the current development of JD.com and draw conclusions. While JD.com continues to invest in maintaining its existing warehousing and logistics advantages, it is not enough for the development of emerging live markets and sinking markets. At this stage, Chinese companies are increasingly going global, and whether they can seize the development of policy and communication technology is a decisive factor in whether self-operated e-commerce can become bigger and stronger. At the same time, it is found that JD.com's marketing model at this stage is lacking in sustained innovation. This paper enriches the post-epidemic era for the development of China's self-operated e-commerce research has positive implications for the development of this industry
\end{abstract}

Keywords: E-commerce; Development Strategy; Innovation

\section{Introduction}

\subsection{Background of China's e-commerce market}

Chinese e-commerce started late compared to developed Western countries. China's e-commerce industry started in the 1990s, but due to China's economy and the degree of market openness and internet penetration, it was not until the 21 st century that China's e-commerce industry entered the right track of development. By the end of 2014, China's e-commerce industry had fully surpassed that of the European Union and Japan. China's e-commerce market is now maturing and there are more and more e-commerce companies competing together. And China's e-commerce market share is gradually expanding. The size of China's online shopping user base will continue to grow at a high rate from 2015 to 2020. 


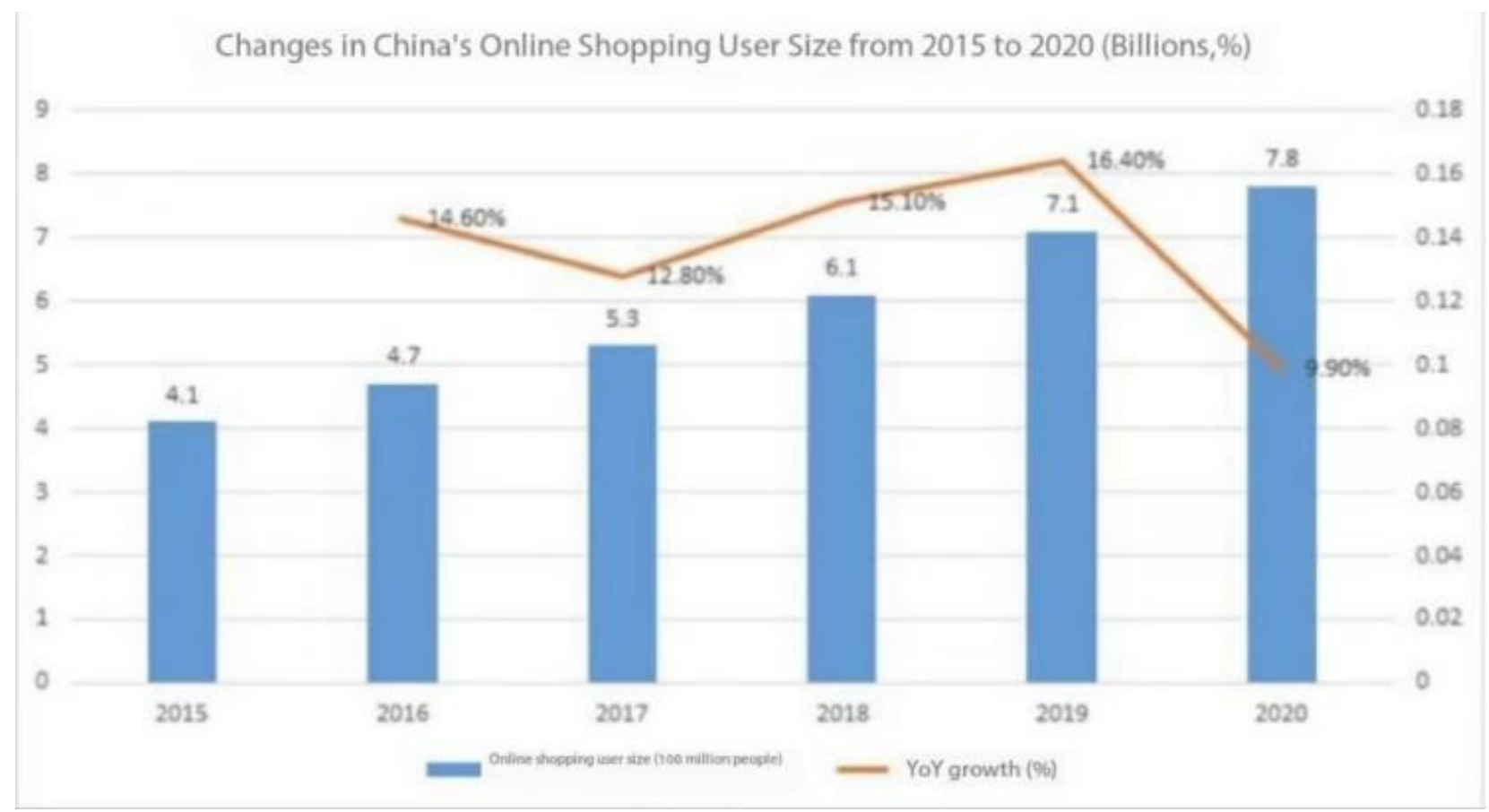

Figure 1. Change in the size of online shopping users in China, 2015-2020 (resources: China Consumers Association).

According to the data in Figure 1, the number of online shopping users in China was 410 million in 2015. In 2016, it was 470 million, an increase of $14.6 \%$ compared with 2015. In 2017, it was 530 million people, an increase of $12.8 \%$ compared with 2016. In 2018, it was 610 million people, an increase of $15.1 \%$ compared with 2017. In 2019, it was 710 million people, an increase of $16.4 \%$ compared with 2018. By 2020 due to the impact of the COVID-19 growth rate slowed to 780 million people year-on-year growth of $9.9 \%$ in 2019. The huge size of China's e-commerce market is not only reflected in the number of users, but also in the scale of online shopping market transactions. In 2015, the transaction scale of China's online shopping market was 3,877.3 billion yuan CNY. By 2020, this figure will increase to $11,760.1$ billion CNY. In 2020, the market size of online shopping in China has tripled to 2015. According to the China Internet Network Information Center, the size of China's Internet users has reached 1.011 billion. The size of China's online shopping users predictably has huge room for growth. And with the development of China's economy and the recovery of the global economy in the post-epidemic era, the potential of China's online shopping market is still huge.

\subsection{Review of the paper}

Regarding SWOT analysis, scholars at home and abroad have been doing research for a long time. Scholars Chen Dongmei, Wang Lizhen and Chen Anni point out that "Diversification strategy, on the other hand, is the basis for the discipline of strategic management to gain realistic legitimacy, and it is after the wide use of tools such as the Boston Matrix and the McKinsey Matrix (GE Matrix), which have significantly improved the efficiency of corporate diversification, that the discipline of strategic management has gained respect from the practical community [1]." Scholar YU Congguo points out that "in the era of "Internet +", the industry boundaries of enterprises are increasingly blurred, and the formulation of strategies based on economic patterns can adapt to the development needs of different industries, enabling strategies to have continuity and inclusiveness [2]." Scholar Wang Baoyi points out that the gradual online retailing in China is affecting the offline retail, service and manufacturing industries. And, this positive impact is gradually expanding [3]. Scholars Chun, Se-Hak ; Kim, JaeCheol state that the higher the convenience of online shopping, the higher the price of goods for online shopping increases or even exceeds the offline price [4]. It is evident that price advantage of B2C ecommerce can hardly be the main driver to continue attracting customers when the logistics grows to a certain scale. 


\section{Data and research method}

\subsection{Data}

Originally founded in 2004, JD. com's main part of business is online retail, and its JD.com Mall is the largest B2C shopping website in China. In May 2014, JD.com Group was listed on the NASDAQ in the United States. JD.com Retail has now completed full category coverage of computer digital, mobile phones, home appliances, consumer goods, fashion, home, fresh food, lifestyle services, industrial goods, etc. JD.com already has more than 9 million SKUs of self-operated products with over 8 million active enterprise customers. By the end of 2020, the number of active buyers in JD.com reached 472 million. This paper combines Chinese and foreign papers, Chinese official database data and JD. com's official financial reports as the main basis of this paper's research.

\subsection{Research method}

SWOT analysis is one of the most commonly used methods for analyzing the competitive environment of a business. It is a macro-level analysis of the company. It contains 4 dimensions which are, Internal Strengths, External Opportunities, Internal Weaknesses and External Threaten. SWOT is a widely used tool by practitioners and market researchers about business marketing and strategy tools. In business, the starting point for strategic planning is usually the grouping of internal and external issue [5].

\section{Results and discussion}

\subsection{JD.com's internal strengths}

JD. com's internal strengths are mainly in logistics, self-operated goods, payments and marketing. Modern warehousing is the node and control center in the logistics and supply chain system [6]. JD.com warehousing and logistics is one of JD. com's core competencies, and JD.com set out to lay out a free warehousing and logistics system at the beginning of its establishment. And until now the warehousing and logistics part is also the key investment and development of the whole JD.com Group. JD.com has been working on improving its infrastructure across China. These infrastructures include large warehousing and logistics centers in regional hubs, regional distribution centers, and collection and delivery sites with self-pickup and courier delivery as the main functions. Currently, JD.com has established seven major logistics centers in Beijing, Shanghai, Wuhan, Shenyang, Guangzhou, Chengdu, and Xi'an. The data shows that JD.com Logistics covers all provinces, autonomous regions and municipalities in China, with a total of 70 cities with large warehouses and 31 cities with front warehouses. By the third quarter of 2020, JD.com will have more than 800 warehouses with a total area of more than 20 million square meters. And the area of new warehouses is larger compared to the area of old warehouses. At present, JD.com Logistics has already covered the logistics system of large goods, cold chain, cross-border, customer service, after-sales, supply chain and express integrated services. Since JD. com's main operation mode is self-operated, and JD.com has a strong independent and autonomous warehousing and logistics infrastructure. This strengthens risk control. 
Volume 16 (2021)

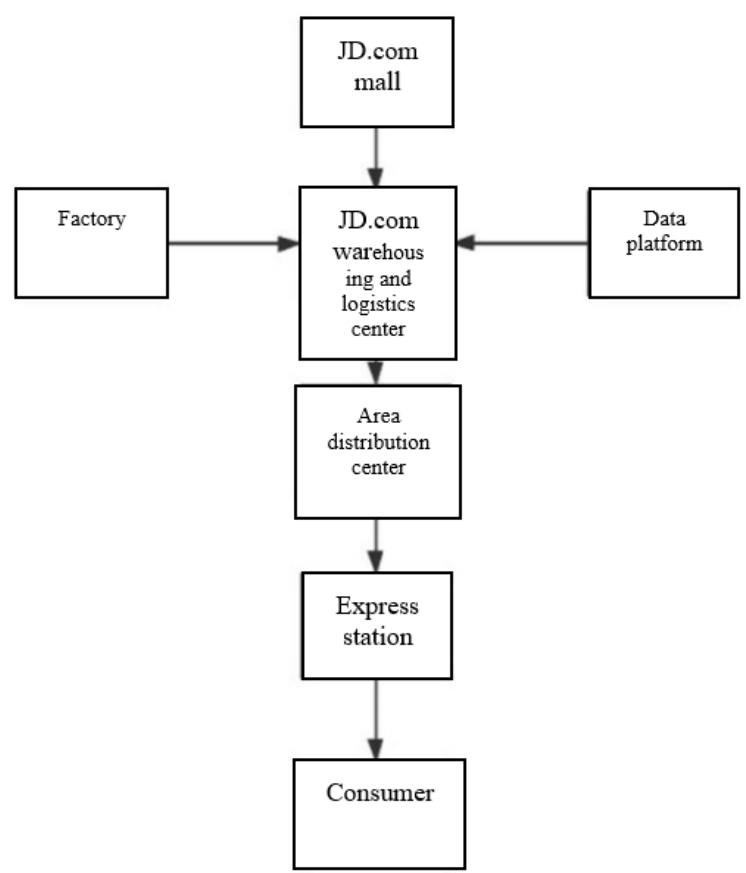

Figure 2. Schematic diagram of JD.com Mall logistics operation (compiled by the author).

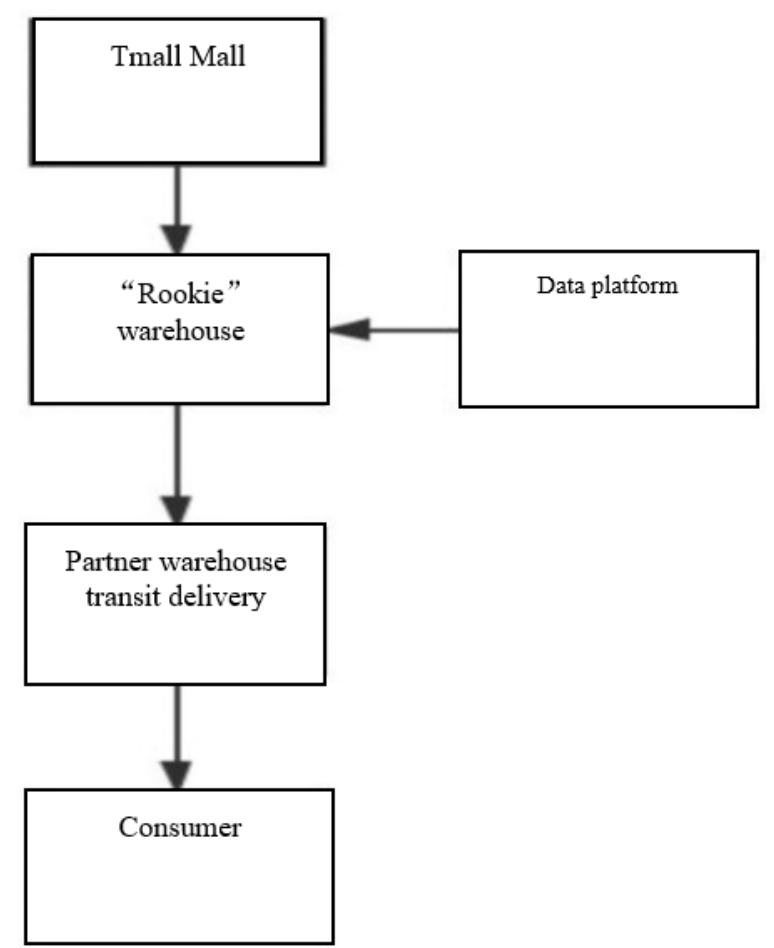

Figure 3. Schematic diagram of Tmall Mall logistics operation (compiled by the author).

As shown in Figure 2 and Figure 3, JD.com and Tmall logistics operation diagram, JD.com can reach $100 \%$ penetration rate on self-operated goods between consumers. JD.com is able to control and complete the whole process from the time the consumer places an order to the delivery of the goods independently. This reduces the risk of damage and loss of goods in transit. Compared to Tmall's logistics system, Tmall only controls the data and the first stage of the goods out of the warehouse, and the goods sorting and transportation and distribution are partially handled by Alibaba's courier partners. Because of the uncertainty of the partner's efficiency, the courier cycle is longer compared to JD.com. At the same time, compared to Tmall's distribution model, JD.com Mall's distribution reduces the logistics risk while also improving the timeliness of the after-sales area, which directly affects customer satisfaction and indirectly affects customer loyalty. JD.com has launched 
211 limited time delivery, extremely fast delivery, same-day delivery in the same city and other time frame commitments. The advantages of JD. com's logistics are not only reflected in the timeliness, but also in the service. JD.com Mall launched a $7 * 24$-hour service line at the beginning of its establishment to ensure that users can respond to the various problems encountered in real time.

In terms of logistics innovation, JD.com is embarking on the transformation from traditional logistics to intelligent logistics. In October 2017, JD. com's first full-process intelligent unmanned warehouse was put into operation. Intelligent logistics overturned the traditional basic labor distribution service [7]. In 2019, JD. com's "Asia One" warehouse in Xi'an was completed and put into trial operation. The warehouse has a floor area of 300,000 square meters and the entire warehouse is highly automated. The storage efficiency is three times higher than that of traditional warehouses. The warehouse can handle 500,000 orders per day [7]. In terms of delivery, JD.com has already started laying out unmanned delivery. According to Globe Newswire on November 22, 2018, the world's first robot-based distribution station was completed and put into operation in Changsha. The distribution station express sorting is highly automated and equipped with a robot docking charging area. The station staff assembles express mail to the JD.com 3.5 generation delivery robot and then the robot automatically delivers the goods to consumers. The robot has autonomous navigation, automatic obstacle avoidance, and traffic light recognition and face recognition capabilities. Each robot can deliver 30 parcels at a time [8].

JD.com has more than 9 million self-operated SKUs. JD.com cooperates directly with various brands, and JD.com acts as a direct agent in the form of, for example, a self-operated flagship store. In the process, JD.com has accumulated a large number of brand suppliers and expanded its customer base. At the same time, JD.com is different from Taobao or eBay and other e-commerce platforms, where merchant body is scattered and the quality of merchants is uneven. JD. com's self-operated goods are more quality assured, which further increases customer loyalty. It is also because JD.com has fewer supply chain links, JD.com can achieve both genuine products and low prices. In terms of shopping platform innovation, JD.com has launched the "JD. Com Loans ". JD.com Loans is a payment method launched by JD.com to pay after spending. Consumers can choose to pay back the full amount after 30 days or in up to 24 installments. This payment method is easy and has a large customer base. JD.com has expanded the sales of its shopping platform through the "JD. Com Loans "credit spending. In terms of marketing innovation, JD.com continues to innovate. Compared to Amazon's big promotions in Western countries, which usually have only two discount seasons per year, JD. com's promotions are more mundane and stronger. JD.com has promotions on every traditional Chinese holiday, and has annual promotions on June 18 and November 11, similar to Black Friday in the US. According to a report by Kaiyuan Securities, JD. com's cumulative turnover for the 2021 "618" promotion increased by $27.7 \%$ year-on-year to CNY343.8 billion. Among the promotional tools, JD.com has introduced cash coupons, discount coupons and pre-sales. JD.com also cooperated with short-video platforms such as "Kwai" to conduct live promotions.

\subsection{JD.com's Internal Weaknesses}

JD. com's internal weakness mainly includes in terms of user stickiness, number of products and business capacity.

User stickiness is user loyalty for e-commerce platforms. According to JD. com's latest financial report, the number of active users in JD.com has reached 471.9 million. But the gap with the number of Chinese online shopping users is huge. There are still a lot of potential users that have not been tapped. Public data shows that JD. com's GMV per capita in 2020 is 5,536CNY. E-commerce platforms such as Alibaba Group and Pinduoduo have already ventured into the sinking market at a very early stage. JD.com is late to the sinking market and new customers in the sink market dilute the GMV growth rate. User stickiness is a core element for survival and growth for the mainland China market. 
In terms of the number of products, JD. com's product range and quantity is relatively small compared to Alibaba Group's Tmall and Taobao platform, and there is little room for users to choose. This reduces the user's buying experience. JD.com is initially a pipeline type of e-commerce, while Taobao is a platform type of e-commerce from the beginning of the creation, although the later Tmall Mall has some self-operated goods but the essence of the platform is still unchanged, which is supplemented by the pipeline of a comprehensive e-commerce platform. Because JD.com has a very strict selection of suppliers for its self-operated goods, the quality can be guaranteed. However, in the process of transitioning from a pipeline to a platform, JD.com has increased the number and variety of merchants to a certain extent but has neglected management. For example, the same brand on JD.com Mall will have many similar store names. For example, self-operated flagship store, official flagship store, official authorized store, and so on. It is easy for consumers to mistake non-selfoperated stores as JD.com self-operated stores, and this is even more obvious after JD.com opens up its logistics to third parties after 2018. If the quality of goods from non-self-operated stores causes consumer dissatisfaction, it is likely that consumers will have negative feelings towards JD.com selfoperated stores. This will lose a large number of users by overdrawing word of mouth in advance. Analysis of data from JD. com's online retailers is shown in the table 1.

Table 1. Key Profitability Indicators of JD.com (Source: Company Financial Reports).

\begin{tabular}{ccc}
\hline JD. com's Profitability Metrics & $2019-2020$ & \\
\hline Key Indicators & 2019 & 2020 \\
Gross margin & 14.63 & 14.63 \\
Net operating margin & 2.06 & 6.62 \\
Total net asset margin & 14.04 & 14.49 \\
Net equity margin & 14.04 & 36.68 \\
\hline
\end{tabular}

JD. com's operating margin is low. JD.com benefits from a highly automated logistics supply system and all figures have improved but overall JD. com's profitability is weak. JD.com needs to focus more on its main business operations while strengthening its technological innovation.

\subsection{External Opportunities for JD.com}

The current external opportunities in the e-commerce market are mainly driven by favorable policies in China and favorable technological innovations in communication networks.

Firstly, China has proposed the idea of "One Belt, One Road", and the total trade between China and the countries along the "One Belt, One Road" from 2013 to 2019 is more than US\$780 million [9]. In the past, China's e-commerce trade targets were mainly European and American countries, just like the traditional offline trade targets. However, due to the influence of international political relations and trade protectionism, European and American countries often adopt high tariffs and strict censorship on Chinese cross-border e-commerce. Along the Belt and Road are more developing countries and some emerging markets such as Southeast Asia. The "Belt and Road" policy provides opportunities for e-commerce companies to develop emerging overseas markets. In the Chinese domestic market segment, JD. com's earnings from 2017 to 2020 show that the company will not reach profitability until 2019. This indicates that JD.com is in the stage of pre-investment until 2019. According to the financial statement of JD.com Company, it is found that JD. com's operating profit in 2019 is 8240.26 million CNY while in $2020 \mathrm{JD}$. com's operating profit is 12414 million CNY. Instead of being negatively affected under theCOVID-19 in 2020, JD. com's profit increased by $50.65 \%$. Marketing model innovation is a large part of the reason for the significant increase in JD. com's operating profit under the epidemic. During the epidemic, Chinese users' online consumption habits accelerated. Live e-commerce became a new, popular marketing model for consumers. As of June 2021, there were 384 million live e-commerce users in China, up 75.24 million year-on-year and accounting for $38 \%$ of the overall internet population. This indicates a large and promising market for live streaming in China. 2020 to 2021 has been able to achieve such high growth mainly because 
of the epidemic, with some Chinese cities adopting closure policies and some cities not even allowing courier vehicles in and out of some neighborhoods. In the process of closing the city, users were influenced by live banding from platforms such as Tik Tok and Kwai and had a revenge spending mentality after the end of the closure. At the same time, the paradigm shift in China's economy has given domestic companies, including e-commerce, a huge opportunity. China's economic cycle model shifted in 2020, according to scholars Hyun Lee and Bojun Cui, who noted that China launched an economic strategy of "international circulation" in the 1980s, which initially lived competitively through China's then-cheap labor force and huge population base. The scale of the economy depended to a large extent on overseas markets. The "external economic circulation" model was the optimal solution, both in terms of the historical context at the time and in terms of the results later [10]. In 2020, China proposed an economic development model based on the "internal cycle" in China and a double cycle in China and abroad. This means that China will undergo a major transformation in the coming period, i.e. reducing its economic dependence on exports from other countries and gradually shifting the main driving force of economic growth to boosting internal consumption. Live ecommerce is a new stage in the evolution of e-commerce development. A large part of the development of live e-commerce is due to the upgrade of mobile communication technology. The development of $5 \mathrm{G}$ has substantially improved the user experience of watching live e-commerce programs. At the same time, the level of traffic tariffs in China has dropped significantly, expanding the customer base of live e-commerce. According to the "48th Statistical Report on the Development of China's Internet" released by the China Internet Network Information Center (CNNIC), as of June 2021, the size of China's Internet users was 1.01 billion, of which $99.6 \%$ used mobile phones to access the Internet, and the Internet penetration rate was $71.6 \%$. This figure exceeds the combined population of North America. However, in terms of the size of urban and rural Internet users, as of December 2020, urban Internet users accounted for $68.7 \%$ of the overall number of Internet users, while rural Internet users only accounted for $31.3 \%$ of the overall number of Internet users, and the difference rate between urban and rural areas narrowed by $4.8 \%$ to $29.4 \% / 70.6 \%$. With this data, the authors found that the Internet penetration gap between urban and rural areas is rapidly narrowing. The sinking market is a very good development direction for e-commerce.

Meanwhile, the popularity of 5G technology in China has boosted the growth of e-commerce. According to data from China Internet Network Information Center, the number of $5 \mathrm{G}$ mobile phone terminal users in China was 365 million as of June 2021. And 5G mobile phones are accelerating in popularity, with 128 million 5G mobile phone shipments in China in the first half of 2021, up 100.9\% year-on-year. 5G's high-speed and high-bandwidth features can solve technical problems that were not possible in the $4 \mathrm{G}$ era if combined with the rapidly developing AI technology. For example, the popularity of mass application of unmanned delivery technology. $4 \mathrm{G}$ era because of bandwidth and network speed constraints, consumers often encounter page lag or even crash in some consumer promotions. $5 \mathrm{G}$ also facilitates the improvement of e-commerce user consumption efficiency and consumption experience.

\subsection{External Environmental Threatens for JD.com}

The e-commerce market is highly competitive, and competitors are not only limited to traditional e-commerce platforms, but also include some emerging cross-border platforms. According to the China Internet Network Information Center, there are 384 million live e-commerce users in China. This represents $38.0 \%$ of the overall Internet users. Short video and live streaming platforms profit model from simple traffic cash breaking circle into the e-commerce industry. For example, Jieyin, Racer and WeChat live streaming platforms have already captured the e-commerce market with high growth rates. According to Zhang Weijia, the top three live e-commerce platforms in China are Jieyin, Taobao and Racer. 2020, these three e-commerce giants occupy $99.7 \%$ of the live e-commerce industry. And in 2020, China's live e-commerce market transaction size has reached 12850 billion yuan compared to 2018, an increase of $589.46 \%$ [11]. What's more remarkable is that Jitterbug Express was mainly food and beverage and personal care when the goods were first involved in the 
e-commerce sector. Taobao Live started to expand its live merchandise business to include home appliances cars and household appliances in 2019. Jitterbug and Racer platforms are already rapidly diversifying their live merchandise categories towards higher value-added goods, such as mobile phones and digital products. This will undoubtedly impact the market share of JD.com Mall with 3C products as the main commodity. The table 2 shows the share of major live e-commerce platforms.

Table 2. Share of major live e-commerce platforms (Source: China Consumers Association).

\begin{tabular}{clccc}
\hline \multicolumn{3}{c}{ E-commerce Share of Live Broadcasting Platform (Unit :\%) } \\
\hline $\begin{array}{c}\text { Live broadcasting platform } \\
\text { Traditional E-commerce Live }\end{array}$ & & Users & Loyal users \\
& Taobao & Live & 68.5 & 46.3 \\
& Tmall & Live & 32.4 & 5 \\
& JD & Live & 23.8 & 3.5 \\
& Pinduoduo & Live & 20.9 & 3.4 \\
Social E-commerce Live & & & & \\
& Tik Tok & Live & 57.8 & 21.2 \\
& Kwai & Live & 41 & 15.3 \\
& Huya & Live & 9.8 & 0.2 \\
& Betta & Live & 12.1 & 0.1 \\
\hline
\end{tabular}

According to the data in Table 2, the traditional Taobao live users accounted for $68.5 \%$ and the loyal users reached $46.3 \%$. Compared with $23.8 \%$ of JD's live users, it has an absolute advantage, while JD live loyal users rate is only $3.5 \%$. The gap in the share of e-commerce shopping platforms between JD.com and Taobao cannot be equalized by JD.com in a short period of time. On the new social live broadcast e-commerce platform, Tik Tok and Kwai live broadcast users both exceed $40 \%$. Tik Tok has even reached $57.8 \%$ and the proportion of loyal users has reached $21.2 \%$. It is foreseeable that because JD.com entered the live industry late or did not pay attention to the rise of live ecommerce, the live broadcast e-commerce market share was seized by other competitors. JD live ecommerce business will be weak in a long period of time in the future.

\section{Conclusion}

Through this paper's SWOT analysis of JD.com Company, it is found that JD. com's innovations are mainly distributed in logistics, financial type of payment and quality of goods and marketing methods. However, JD.com ignored the cutting-edge new live streaming market, although after 2018 JD.com started remedial measures to enter the field of live streaming. But the data shows that the rate of using users, especially loyal users, is still very low. At the same time, the self-operated strategy is a double-edged sword, improving the quality assurance of goods and JD.com self-operated goods in consumer reviews while also somewhat reducing the richness of JD. com's goods. At present, the transformation of JD.com from a pipeline to a platform has not been completely successful JD.com still needs to continue to open up its platform to attract more third-party merchants while strengthening supervision. The lack of commodity richness will also directly affect user loyalty. JD.com has invested a lot of money to build its own logistics network, but with the expansion of the scale of basic logistics facilities. The operation and management costs are bound to increase, so JD.com should take advantage of the $5 \mathrm{G}$ policy to increase the automation of its logistics network. JD.com should actively participate in the "One Belt, One Road" policy to develop overseas markets, and for the domestic JD.com should invest more in, for example, JD. com's "Jingxi" community group-buying platform to develop and develop the sink market. At the same time, for the emerging competitors such as social live platform for JD.com traditional main market, digital products and other areas of market share erosion, JD.com should be vigilant to strengthen the innovation of sales methods. 
Data show that the traditional Taobao live users accounted for up to $68.5 \%$ and loyal users reached $46.3 \%$. Compared to JD.com live users $23.8 \%$ has an absolute advantage, while JD.com has only $3.5 \%$ of loyal live users. The gap between JD.com and Taobao in the share of the e-commerce shopping platform is not JD.com can catch up in a short time. The new social live e-commerce platform, Jitterbug, Racer live users are more than $40 \%$, Jitterbug has even reached $57.8 \%$ and loyal users accounted for $21.2 \%$. It is predictable that JD.com because of the late entry into the live industry, or for the rise of live e-commerce out of the lack of attention led to live e-commerce market share by other competitors, JD.com in the future for a long time live e-commerce business will be in a weak position.

\section{References}

[1] CHEN Dongmei, WANG Lizhen, CHEN Anni. (2020). Digitalization and Strategic Management Theory - Review, Challenges and Prospects, Management World, no. 220-236.

[2] SHE Congguo. (2015). Strategic management innovation of enterprises in the era of "Internet+", Communication Enterprise Management, no. 28-29.

[3] WANG Baoyi. (2017, April). The evolution, competitive situation and development trend of China's e-commerce e-tailing industry, China Circulation Economy, no. 25-34.

[4] Chun\& Kim, J.-C.S.-H. (2005). pricing strategies in B2C electronic commerce: analytical and empirical approaches. Decision Support Systems, no, 375-388.

[5] Marilyn M. HelmsJudy Nixon\&. (2010). Exploring SWOT analysis - where are we now: A review of academic research from the last decade. Journal of Strategy and Management, no, 215-?

[6] Wang, X., Li, M., Zhang, X., \& Zhang, Y. (2020). Process Analysis of Warehouse Logistics Information System. IOP Conference Series. Earth and Environmental Science, 526(1), 12210-. https://doi.org/10.1088/1755-1315/526/1/012210

[7] HAO Li, ZHANG Yuxi. (2020(12)). Study on the current situation and countermeasures of the development of intelligent logistics in Jingdong [J]. China Storage and Transportation, no, 85-88.

[8] Globe. $(2019,26(01))$. The first robotic intelligent delivery station opened [J]. Instrument user, no, 89.

[9] CAI Fang. (June 04, 2021). Promoting "One Belt, One Road" construction to go deeper and more practical. Economic Daily.

[10]LI Xian, CUI Bojun. (2020, 46(06)). Live electric business in the perspective of domestic economic cycle $[\mathrm{J}]$. Thought Line, no, 56-63.

[11]ZHANG Weijia. (September 08, 2021). China's live e-commerce industry market status and competitive landscape analysis in 2021. 2021 GMV is expected to exceed 2,300 billion yuan. Foresight Industry Research Institute 